\title{
Do People Differentially Remember Cheaters?
}

\author{
Pat Barclay \\ McMaster University

\section{Martin L. Lalumière \\ Centre for Addiction and Mental Health, and University of Toronto}

The evolution of reciprocal altruism probably involved the evolution of mechanisms to detect cheating and remember cheaters. In a well-known study, Mealey, Daood, and Krage (1996) observed that participants had enhanced memory for faces that had previously been associated with descriptions of acts of cheating. There were, however, problems with the descriptions that were used in that study. We sought to replicate and extend the findings of Mealey and colleagues by using more controlled descriptions and by examining the possibility of enhanced altruist recognition. We also examined whether individual differences in cheating tendencies were related to cheater and altruist recognition. In the first experiment, 164 undergraduates saw 40 faces that were paired with character descriptions representing the categories cheating, trustworthiness, altruism, or neutral, for individuals who had either low or high social status. One week later participants reported which faces they recognized from the previous week (among 80 faces). Overall, the results failed to replicate the findings of Mealey and her colleagues, as there was no enhanced memory for cheaters. In addition, there was no enhanced memory for altruists, and no effect of participants' cheating tendencies. A second experiment using a slightly different methodology produced similar results, with some evidence for enhanced memory for altruists.

KEY WORDS: Altruism; Cheater recognition; Cheating detection; Memory

$\mathrm{I}_{\mathrm{b}}^{\mathrm{n}}$ ndividuals who follow a strategy of reciprocal altruism can be very successful because they reap the benefits of mutual cooperation (Axelrod and Hamilton

Received March 22, 2004; revisions requested August 10, 2004; revised version accepted January 11,2005

Address all correspondence to Pat Barclay, Department of Neurobiology and Behavior, W313 Mudd Hall, Cornell University, Ithaca, NY 14853-2702.Email: pjb46@cornell.edu

Human Nature, Spring 2006, Vol. 17, No. 1, pp. 98-113.

$1045-6767 / 98 / \$ 6.00=.15$ 
1981; Trivers 1971). The maintenance of reciprocal altruism over evolutionary time likely requires that individuals avoid exploitation by (1) detecting instances of nonreciprocation (cheating detection) and (2) remembering who has reciprocated and who has not (cheater recognition), and avoiding cooperative interactions with nonreciprocators (Trivers 1971). Cosmides (1989) proposed the existence of cheating detection mechanisms in the human brain that are specifically designed to help recognize violations of social contract rules. There has been much research on cheating detection, and it has been referred to as one of the flagship results in evolutionary psychology (Fodor 2000). This research has been conducted primarily with the Wason selection task, which is prone to method artifacts based on the type of language used, such that people might not be actually detecting cheating but responding to other features of the Wason tasks (Fodor 2000). There has been very little research on people's special ability to remember or recognize individuals who have demonstrated a willingness to cheat.

\section{CHEATER RECOGNITION}

Mealey, Daood, and Krage (1996) used face recognition to test people's ability to remember cheaters. They paired various pictures of faces with descriptive sentences representing the categories of cheater, trustworthy, or neutral. Participants first rated the attractiveness of the pictures under the guise of test-retest reliability, and then returned one week later to identify the faces they remembered. Mealey and colleagues found that participants were better at remembering faces that had been paired with descriptions of cheaters (but only if the cheaters were of low status), and that males were more biased toward remembering cheaters than females were.

This method is potentially quite useful as a research tool, but it is also prone to subtle methodological difficulties. For instance, descriptions of particular behaviors may be memorable for reasons other than the fulfilling or breaking of a social contract. In Mealey et al.'s (1996) study, one could argue that the salience of the actions of cheaters was greater than the salience of actions described in the other categories: Some cheaters were not only cheaters in terms of a social contract, but threatening or physically dangerous individuals such as thieves or child molesters. Another problem was the correct assignment of particular actions to the different categories of social contract behavior and the heterogeneity of behaviors within a particular category. In Mealey et al.'s study, the trustworthy individuals included altruists (volunteer Big Brothers), people reporting potential problems at work (such as an oil leak or suspicious-looking people), and individuals exposing other colleagues. These do not cleanly fit the category "trustworthy."

We know of only three studies that have attempted to replicate Mealey et al.'s (1996) results. In an unpublished paper, Baron and Burnstein (2002) found that participants were better at remembering faces paired with character information than faces paired with neutral information. The valence of character information (positive vs. negative) did not have an effect on memory accuracy. The time be- 
tween exposure to the pictures and the recognition task was 10 minutes, compared with one week in Mealey et al.'s study.

Oda (1997) asked participants to imagine they were in a prisoner's dilemma game and to choose a constant strategy of cooperation or defection. They then rated the attractiveness of male and female faces described as cooperators or defectors in the game. On a recognition test one week later, male defectors were remembered better than male cooperators, but there was no effect for female faces. Female defectors and cooperators were remembered about as often as the faces of male defectors, and subjects' sex and own strategy had no effect.

Finally, Chiappe et al. (2004) asked participants to categorize people as either cheaters, cooperators, or neither, based on a description of their behavior. They then asked them, immediately after, which faces they remembered. Faces of people categorized as cheaters were more often recognized than faces categorized as cooperators or as neither. It seems, however, that the "degree" of cheating by the cheaters in some descriptions was much higher than the "degree" of cooperation by the cooperators, which could easily have increased the salience of the cheaters. Together these three studies provide marginal evidence for the presence of a cheater-recognition mechanism.

\section{ALTRUIST RECOGNITION}

Kiyonari, Tanida, and Yamagishi (2000) argue that a focus on cheater-detection has overshadowed research on other important aspects of social exchange. As mentioned by Brown and Moore (2000), being able to detect altruists and then cooperating only with them may be a way to solve the problem of being exploited in social interactions. Other researchers have suggested that paying attention to altruists may be beneficial because altruism may be a signal of phenotypic quality or willingness to cooperate (e.g., Barclay 2004; Smith, Bowles, and Gintis 2000; Zahavi and Zahavi 1997). Thus, humans may be expected to be good at altruism detection and altruist recognition, and Brown and Moore indeed found evidence for altruism detection using the Wason selection task. We know of no studies investigating enhanced memory for altruists.

\section{CHEATING TENDENCIES AND CHEATER RECOGNITION}

It is not known whether a person's tendency toward cheating or cooperating affects his or her detection of particular social contract behavior or recognition of particular individuals. The only study addressing this question is the aforementioned study by Oda (1997), which had participants choose a cheating or cooperating strategy in multiple one-shot prisoner's dilemma games. Their choice was unrelated to subsequent recognition of cheaters (or cooperators) one week later.

Psychopaths are individuals who may be expected to behave differently in social contract situations. Although psychopaths account for only 15 to $25 \%$ of incarcer- 
ated males, they account for a disproportionate amount of crime and violence in society (Hare, Forth, and Strachan 1992). Psychopaths lack many social emotions, such as empathy, guilt, shame, and remorse, that may underlie reciprocal altruism. They are very effective at manipulating others, so much so that they have been described as leading parasitic lifestyles. In a study of 47 men recruited from employment agencies, Seto, Khattar, Lalumière, and Quinsey (1997) found that scores on a measure of psychopathy were related to a tendency to deceive others in both sexual and nonsexual contexts. Psychopaths are often considered "cheaters" because they often defect on people after signaling a willingness to cooperate. This "cheater" or "defector" strategy may have evolved as an alternative life-strategy via frequency-dependent selection (Mealey 1995; Lalumière, Harris, and Rice 2001).

There are no published studies examining cheating detection or cheater recognition among psychopaths, so it is not currently known whether psychopaths have the same social exchange algorithms that all humans are purported to have. On the one hand, we might predict that psychopaths have the same mechanisms as other humans, because psychopaths need to protect themselves from being cheated just like anyone else. On the other hand, if cheating detection occurs because people are detecting behaviors that deviate from their own or from the norm, then psychopaths might not be as good at detecting cheating (or remembering cheaters) because they are cheaters themselves.

Another possibility is that psychopaths are poor at cheater-detection tasks because they do not recognize (or care about) the breaking of a social contract by themselves or others. One could predict, however, that psychopaths are particularly good at altruism detection and altruist recognition because altruistic acts are different from the social norm and from the behavior of psychopaths, and altruists are good targets for psychopaths to exploit.

The current study is a partial replication of the Mealey et al. (1996) study, adding "altruists" as a fourth target category of social exchange, and it attempts to ensure that all character descriptions fit their category properly and have a similar salience. We also measured psychopathy (or cheating tendency) in order to see whether individuals scoring high on psychopathy would be better at remembering altruists than individuals scoring low on psychopathy. Our population of undergraduates is unlikely to contain true psychopaths, but studies of psychopathic tendencies in noncriminal populations can be informative about the role and significance of these tendencies (e.g., Lalumière and Quinsey 1996; Seto et al. 1997).

\section{EXPERIMENT ONE}

\section{Method}

Participants. Participants were enrolled in a first-year undergraduate psychology course and received experimental credit for their participation. Ninety females and 96 males participated in the first week; 83 females (mean age $=20.1 \pm 0.42$ years) 
and 81 males (mean age $=19.2 \pm 0.11$ years) of mixed ethnicity returned the second week and completed the second half of the experiment.

Stimuli. Photographs of Caucasian males were obtained from the Internet (mostly from personal ads) and were trimmed (with Adobe Photoshop) to remove any background or clothing cues that might make some photos more memorable than others. Sixty-five pictures were pre-rated for attractiveness by 12 other volunteers from the same undergraduate population, and the 40 pictures that were within one standard deviation of the mean were chosen for the experiment.

Each photo was paired randomly with a fictional description that gave information on the person's character and socioeconomic status. To control for the possibility that something in the descriptions other than character or status would affect memory, five areas of occupation were balanced over the experimental conditions (arts, construction, medicine, science, and sports). For each of the five occupational areas there was one description from one of eight categories using a $2 \times 4$ design with two levels of status (high and low) and four levels of character information (history of cheating, history of trustworthiness, history of altruism, and neutral or irrelevant information), making 40 descriptions in total. Cheaters were individuals who had broken a social contract; trustworthy individuals had fulfilled a social contract; altruists had gone above and beyond what was expected in a social contract; and neutral individuals had information that had nothing to do with a social contract. ${ }^{1}$

Each description was rated by 12 volunteers from the same population on each of the social contract dimensions on scales of 1 to 7 . As expected, descriptions of cheaters were rated as worse cheaters than other descriptions, trustworthy descriptions were rated as being more trustworthy, and altruistic descriptions were rated more altruistic (there was some overlap between the degree of altruism of the trustworthy and altruistic descriptions). The degree of cheating in the cheating descriptions ( mean $=6.11$, s.d. $=0.53$ ) was not different from the degree of trustworthiness in the trustworthy descriptions ( mean $=6.06$, s.d. $=0.57$ ) or the degree of altruism in the altruistic descriptions ( $\operatorname{mean}=6.27$, s.d. $=0.36, F<1$ ), suggesting that the manipulations of character were of equal salience.

The 40 faces were presented to the participants on a screen in a randomly generated order that was different for every group of participants, and each participant within the group read the descriptions in a booklet in one of 16 randomly generated orders. Thus, there was an equal chance of each description appearing with each face.

Psychopathy Measures. The Levenson psychopathy scale (Levenson, Kiehl, and Fitzpatrick 1995) is a 26-item questionnaire designed to be similar in content to Hare's Psychopathy Checklist (PCL-R; Hare 1991) and particularly relevant for use with university students. Each question is scored from 1 (disagree strongly) to 4 (agree strongly). The items address antisocial attitudes and behaviors. Higher scores indicate a higher level of psychopathic tendencies. 
The Childhood and Adolescent Taxon Scale self-report version (CAT-SR; Seto et al. 1997) consists of eight items that measure early behavioral problems associated with adult psychopathy (Harris, Rice, and Quinsey 1994). Each of the eight items is scored as absent (0), some indication (1), or present (2), and an average of the eight items is taken. Both of these measures have good construct validity.

Procedure. The experiment was run in one large classroom or one of three seminar rooms and consisted of two sessions separated by one week. In the first session, participants were told that we were studying the test-retest reliability of attractiveness ratings. Using a 7-point Likert scale, participants rated how attractive the faces were to them, and then they rated how attractive the targets were to them based on both the picture and the description. Each face was presented for 15 seconds. After rating the 40 faces, participants filled out the Levenson psychopathy scale and CATSR, and answered some biographic questions. The second session was exactly one week later, and participants returned to view (without descriptions) the same 40 faces plus 40 new faces taken from similar sources (all presented randomly). Participants were asked how attractive each face was, and whether or not they remembered it. Memory for faces was tested both with a yes/no question and a scale of 1 ("definitely did not see him") to 9 ("definitely did see him") with 5 ("I guessed randomly") in the middle. The study received approval from the McMaster University Research Ethics Board.

Statistical Analysis. Participants' scores on the Levenson psychopathy scale and CAT-SR were converted to $z$ scores within each sex and averaged to produce a psychopathy score relative to the other participants of the same sex. Based on this score, participants were grouped into tertiles within each sex (the results were the same when participants were grouped into halves or quartiles). We analyzed the data using a $2 \times 3 \times(2 \times 4)$ General Linear Model mixed design, with sex and psychopathy group as between-subject factors and description status and character as within-subject factors.

\section{Results}

Scores on Psychopathy Measures. Scores on the Levenson psychopathy scale ranged from 30 to 85 (out of 96), with a median of 50 and a mean of 51.2. Scores on the CAT-SR ranged from 0 to 1.38 (out of 2), with a median of 0.13 and a mean of 0.23 . As one would expect, males scored higher on both the Levenson psychopathy scale and the CAT-SR than women, $t_{162}=3.34$ and $3.13 ; p<.005$, respectively. Individual scores on these two scales were significantly correlated in men, $r_{79}=$ $0.419, p<0.001$, but not in women, $r_{81}=0.08$, n.s.

Attractiveness. Despite selecting pictures for a restricted range of attractiveness, the one-week test-retest correlation of the attractiveness ratings of the faces was 
quite high: average $r$ across all 40 descriptions $=0.60$, all $p$ values $<0.001$. The descriptions caused significant changes in the attractiveness ratings of the targets in the first session. There were significant effects of character, $F_{3,474}=408.18, p<$ 0.001 ; status, $F_{1,158}=25.65, p<0.001$; and the interaction between them, $F_{3,474}=$ $29.87, p<0.001$, on the amount of change in attractiveness ratings after participants read the descriptions. Cheaters decreased in attractiveness, faces with neutral descriptions increased slightly in attractiveness, trustworthy individuals increased more, altruists increased the most, and the differences between them were all highly significant: all $F$ values $>60$, all $p$ values $<0.001$. High-status descriptions caused greater changes in attractiveness than low-status descriptions.

There was a significant sex $\times$ psychopathy $\times$ character interaction, $F_{6,474}=2.48$, $p<0.05$, and further analysis revealed a significant psychopathy $\times$ character interaction in males, $F_{6,234}=2.87, p=0.01$, but not in females, $F_{6,240}=0.20$, n.s. Examining this interaction in males revealed that descriptions involving cheating had less of an effect on the attractiveness ratings of the faces if the raters were males who ranked high on the psychopathy measures than if the raters had low or moderate rankings, $F_{2,78}=4.76, p=0.01$, but there was no such effect for descriptions involving trustworthiness, $F_{2,78}=1.24$, n.s.; altruism, $F_{2,78}=0,77$, n.s.; or neutrality, $F_{2,78}=0.71$, n.s. In fact, there was a negative correlation between participants' psychopathy scores and the amount of change in attractiveness of pictures of cheaters after participants read the description, $r_{162}=-0.26, p=0.001$, indicating that individuals scoring relatively high on psychopathy tended to "dislike" the cheaters less than individuals scoring low on psychopathy did.

The effects of descriptions on face attractiveness, however, were ephemeral. When the pictures were rated one week later, there was only a marginally significant difference between the four types of character descriptions, $F_{3,474}=2.22, p=0.09$, although this difference followed approximately the same pattern (cheaters $<$ neutral $<$ trustworthy $<$ altruists). Status no longer had an effect, $F_{1,158}=1.36$, n.s., nor did the interaction between status and character, $F_{3,474}=1.27$, n.s.

Face Recognition. On the dichotomous yes/no measure for recognition, participants recognized more faces in this experiment than in the Mealey et al. (1996) study; a mean of 29 out of 40 original faces was remembered, in contrast with 16 out of 36 in the 1996 study: $\mathrm{X}^{2}{ }_{1}=6.2, p<.05$. False alarms were low: mean $=3.5$, s.e. $=0.26$, median $=2$, mode $=1$. The mean number of faces recognized in each category is depicted in Figure 1. Females remembered more faces than males, $F_{1}$, ${ }_{158}=9.65, p=0.002$, but there were no other significant differences in the number of faces remembered (all $p$ values $>0.15$ ). Neither character, $F<1$, nor status, $F<$ 1 , had a significant effect, nor did the interaction between them, $F<1$, or the interaction with psychopathy, $F_{6,474}=1.50, p=0.18$. Using planned contrasts, cheaters, $F_{1,158}=1.56, p=0.21$, altruists, $F<1$, or trustworthy individuals, $F<1$ were not remembered more often than neutral individuals. The analysis of the continuous measure of face recognition produced essentially the same results: Females were 
more sure that they remembered faces than males, $F_{1,158}=10.51, p=0.001$, but there was no difference for recognition of cheaters, neutral individuals, trustworthy individuals, or altruists, nor did status or psychopathy have any effect.

There was no significant correlation between the effect each description had on the attractiveness of its corresponding picture and the likelihood of the face being remembered a week later, $r_{s}(38)=0.22, p=0.18$. The same result was obtained for descriptions of cheaters only, $r_{s}(8)=0.32, p=0.37$. Similarly, there was no significant correlation between the salience or intensity of cheating in the cheating descriptions (as rated by the 12 independent raters) and the likelihood of the faces being remembered, $r_{s}(8)=-0.12, p=0.75$.

\section{Discussion}

Contrary to our expectations, there was no enhanced memory for the faces of cheaters or altruists. Thus, this study failed to replicate Mealey et al.'s (1996) findings that low-status cheaters were remembered better than other individuals. The participants' cheating tendencies had no effect on face recognition. In the following we discuss possible reasons for the failure of this study to replicate the results of the earlier study.

The descriptions used in this experiment differed from the descriptions used in Mealey et al. (1996). We attempted to standardize our descriptions so that only

Figure 1. Mean number (and standard errors) of faces recognized (out of five) in each of the eight categories of description, averaged across all respondents of each sex.

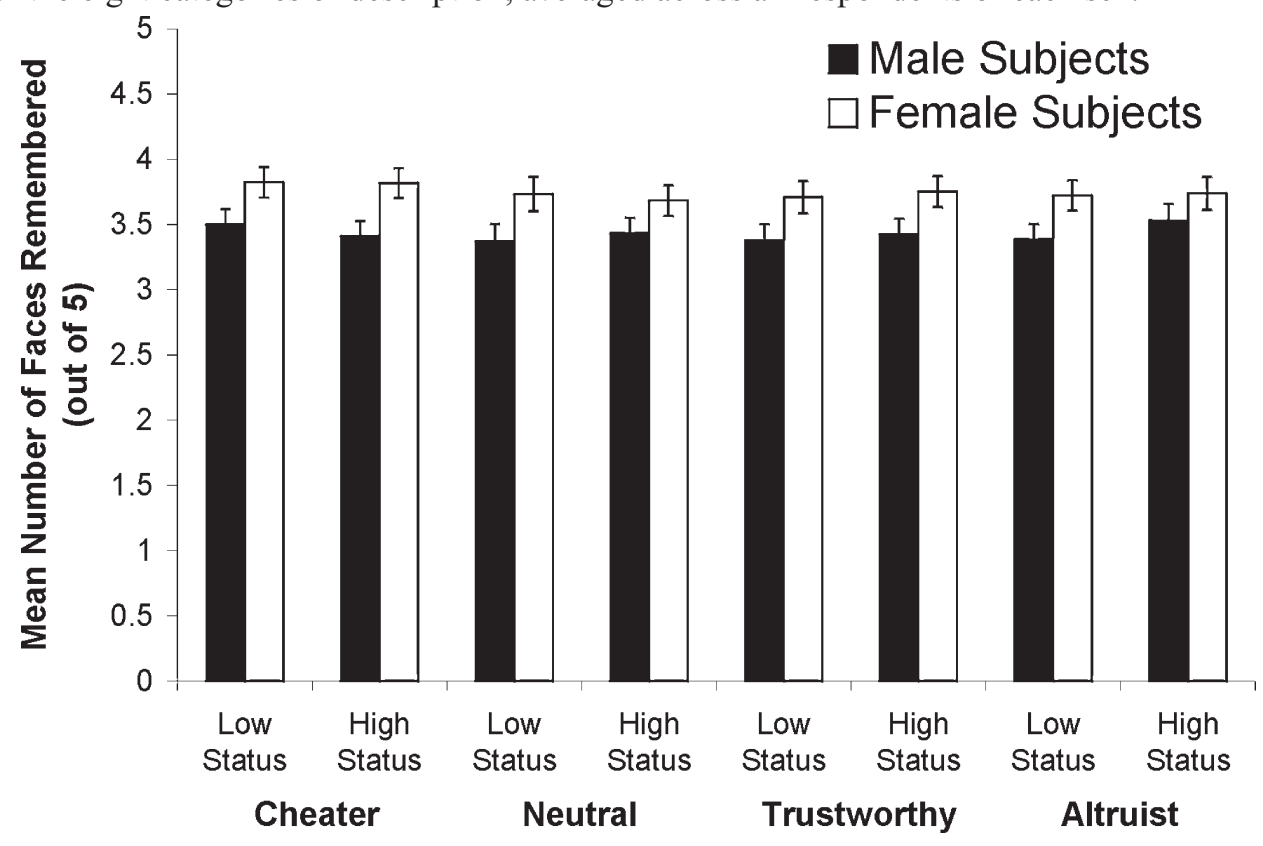


character varied within general occupational domains, and so that other unique features of the descriptions would not make some descriptions more memorable than others. Also, our descriptions were rated as being of equal salience. In the Mealey et al. study, some of the cheaters were threats or dangers in addition to being cheats, and some of the trustworthy individuals may not have been interpreted as being trustworthy. Much of the cheating/cheater detection literature deals with experiments that rely heavily on language, and the results can be easily affected by slight changes in wording (e.g., Cosmides and Tooby 1992; Cummins 1999; Gigerenzer and Hug 1992). As mentioned by Fodor (2000), it is possible that observations of biased cheating detection and cheater recognition are artifacts of the materials used. This said, we must also look at possible weaknesses in the methods used in this experiment that may have prevented the observation of biased memory for cheaters.

One possibility is that participants were not paying attention to the descriptions of character, thereby preventing the association of particular characters with particular faces. Contrary to Mealey et al. (1996), who presented both the faces and the descriptions on the same medium (paper booklet), in this experiment the faces were projected on a big screen, and the descriptions were presented on a booklet. In fact, some of the participants commented after the experiment that they did not pay much attention to the descriptions. The analysis of attractiveness ratings suggests, however, that our manipulations of character had some effect. There was a change in the attractiveness of the targets in the expected direction after participants read the descriptions. It is quite possible, of course, that these effects represent demand characteristics, with participants rating the targets according to how they think descriptions should be rated. Regardless, these results suggest that participants were paying attention to the descriptions.

It is intriguing that ratings by males with high psychopathy scores were not as affected by descriptions of cheating as were ratings by males with low psychopathy scores. This could be because individuals who score high on psychopathy do not realize that certain behaviors are socially undesirable, perhaps because they themselves perform such acts. Individuals scoring very low on psychopathy (and women in general) may be particularly motivated to detect and remember those who cheat, and may perceive them as less attractive persons. Alternatively, individuals scoring high on psychopathy may be less susceptible to demand characteristics. At the very least, the change in target attractiveness supports the notion that participants were paying attention to the descriptions and that cheating was perceived by some as undesirable.

Another issue is age. In order to have descriptions similar to those of Mealey et al. (1996) and to match occupations across character type, our descriptions were of people who would have been older than the participants, and the faces were chosen to match that age range (several participants commented on the age of the targets). Therefore, the faces may not have been relevant enough to the participants for them to pay much attention to the descriptions. Relevance to participants may have been 
the reason why females remembered more faces than males, as the faces depicted males. However, this issue is unlikely to have caused a difference between our results and that of Mealey et al., because the males depicted were older in both studies.

Other methodological differences between this experiment and the Mealey et al. (1996) experiment also could have caused a failure to replicate the results. In the current experiment, faces were presented for 15 seconds each. This would eliminate any effects caused by participants choosing to look at some faces longer than others, which may be the mechanism by which people encode some faces better than others. It could also have caused a ceiling effect, given that more faces were remembered in this study than in Mealey et al.'s study. However, a ceiling effect is unlikely because there was a significant difference in face memory between males and females, and this difference was larger than any differences caused by descriptions. Also, the recognition rates were not very different from the 32 out of 48 found by Chiappe et al. (2004), and they encountered no ceiling effect. It is possible, however, that the standardized time may have hurried some of the participants whose first language was not English, such that the manipulations in the descriptions were not as effective for those participants.

We conducted a second experiment to deal with some of these methodological limitations and differences. In this experiment, both the pictures and the descriptions were presented contiguously on a computer screen, and participants could view and rate them at their own pace.

\section{EXPERIMENT TWO}

\section{Method}

Participants. Participants were enrolled in a first-year undergraduate psychology course and received experimental credit for their participation. Forty females and 21 males participated the first week; 32 females (mean age $18.7 \pm 0.59$ years) and 17 males (mean age $19.7 \pm 0.24$ years) of mixed ethnicity returned the second week and completed the second half of the experiment.

Stimuli. The pictures and descriptions were the same as those used in Experiment One.

Procedure. The procedure was the same as in Experiment One, except that participants were seated at individual computers in a small room and saw the stimuli on their computer screens at their own pace. Picture and description orders were randomly determined for each participant. Looking times were recorded via the computer in both weeks, starting from when the picture and description appeared to when the participant indicated his/her response on the keyboard. The data were analyzed in the same way as in Experiment One. Once again, psychopathy had no 
effect, so for simplicity the data are presented below without the psychopathy variable.

Results

Attractiveness. Once again, one-week test-retest correlation of the attractiveness of the faces was high: average $r$ across all 40 descriptions $=0.69$, all $p$ values $<0.001$. The character descriptions caused significant changes in the attractiveness of the targets in the first session. There were significant effects of character, $F_{3,141}$ $=102.30, p<0.001$; status $F_{1,47}=5.55, p=0.023$; and the interaction between character and status, $F_{3,141}=12.48, p<0.001$. Cheaters decreased in attractiveness, faces with neutral descriptions increased slightly in attractiveness, trustworthy individuals increased more, altruists increased the most, and the differences between these were all highly significant: all $F$ values $>19$, all $p$ values $<0.001$. High-status descriptions caused greater changes in attractiveness than low-status descriptions. These effects were also ephemeral-descriptions had no effect on attractiveness one week later, $F<1$, n.s., and the rank order was not preserved.

Looking Time. There were significant differences in looking time in the first week between descriptions with different types of character information: $F_{3,141}=5.72, p$ $=0.001$. Trustworthy individuals tended to be looked at longer (mean $=7.72 \mathrm{sec}$ onds, s.e. $=0.37)$ than cheaters $($ mean $=7.12$ seconds, s.e. $=0.34)$, altruists (mean $=7.08$ seconds, s.e. $=0.29)$, or neutrals $($ mean $=6.81$ seconds, s.e. $=0.34)$. There were no significant differences in looking time the second week between cheaters $($ mean $=7.63$ seconds, s.e. $=0.29)$, neutral individuals $($ mean $=7.60$ seconds, s.e. $=$ 0.27 ), trustworthy individuals (mean $=7.45$ seconds, s.e. $=0.27$ ), and altruists (mean $=7.32$ seconds, s.e. $=0.28): F_{3,141}=1.45$, n.s.

Face Recognition. Using the dichotomous yes/no measure for face recognition, 30 out of 40 faces were remembered on average (compared with 29 out of 40 in the first experiment). False alarms were low: mean $=3.0$, s.e. $=0.5$, median $=1$, mode $=0$. There was no effect of description character, $F_{3,141}=1.22$, n.s.; status, $F_{1,47}=$ 0.08 , n.s.; or the interaction between the two: $F_{3,141}=1.34$, n.s. Using planned contrasts, cheaters, $F<1$; altruists, $F_{1,47}=1.82, p=0.18$; and trustworthy characters, $F<1$, were not remembered more often than neutral individuals.

There was no effect of participant sex on face recognition, $F_{1,47}=1.47$, n.s., nor did participant sex interact significantly with any of the other variables: character, $F_{3,141}=0.77$; status, $F_{1,47}=3.08$; or the three-way interaction, $F_{3,141}=1.07$ (all n.s.). The mean number of faces remembered in each category is displayed in Figure 2 .

There was no significant correlation between the effect each description had on the attractiveness of its corresponding picture and the likelihood of the face being remembered one week later-for all descriptions, $r_{s}(38)=-0.18, p=0.28$, or for 
Figure 2. Mean number (and standard errors) of faces recognized (out of five) in each of the eight categories of description, averaged across all respondents.

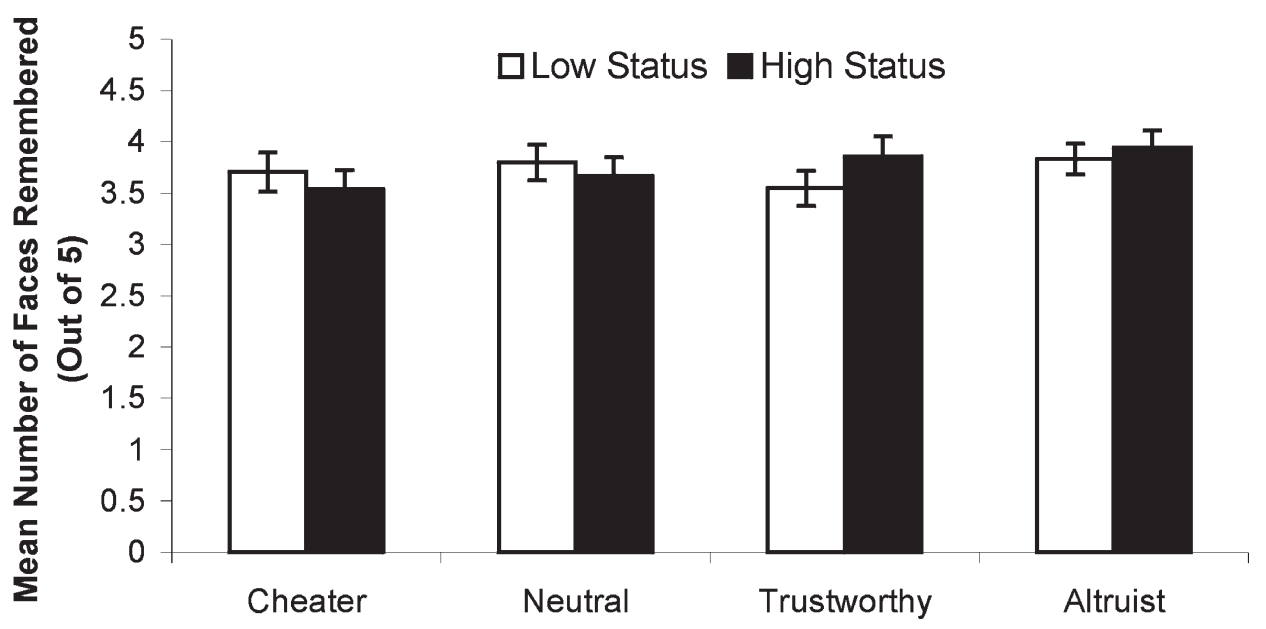

cheating descriptions alone, $r_{s}(8)=-0.38, p=0.28$. This indicates that the descriptions' effects on attractiveness did not have a significant impact on face memory and, if anything, made them less memorable in this experiment. Similarly, there was a slight (but not significant) negative correlation between the rated intensity of cheating in the cheating descriptions (as rated by the independent raters) and the likelihood of the faces associated with them being remembered: $r_{s}(8)=-0.33, p=$ 0.36 . Finally, there was no significant correlation between the rank orders of how much the character descriptions affected the memory of faces in the two experiments: $r_{s}(38)=0.17, p=0.29$.

Using the continuous scale of face memory, there was a significant effect of description character, $F_{2.62,123.33}=2.87, p=0.046$; altruists were more likely to be remembered than cheaters, $F_{1,47}=7.98, p=0.007$, neutrals, $F_{1,47}=5.09, p=0.029$, and trustworthy individuals, $F_{1,47}=6.15, p=0.017$ (Figure 3 ). There were no other significant differences, nor were there any effects of status and participant sex (or their interaction). One possible problem with this analysis is that responses on the 1-9 scale were not normally distributed, and sphericity assumptions were violated (although this was corrected by using the conservative Greenhouse-Geisser correction on the degrees of freedom). If we only consider those faces that participants were very sure that they remembered ( 8 or 9 on the 9-point scale) as successful recognition and count other responses as misses, the trend of altruist recognition was even more pronounced and significant: Altruists (mean $=5.8$ out of 10 , s.e. $=$ $0.35)$ were more likely to be remembered than neutral individuals ( mean $=5.1$, s.e. $=0.34), F_{1,47}=6.25, p=0.016$; trustworthy individuals $($ mean $=5.0$, s.e. $=0.39$ ), $F_{1,47}=5.26, p=0.026$; or cheaters $($ mean $=4.9$, s.e. $=0.36), F_{1,47}=8.96, p=0.004$. The interaction between character and status was significant, $F_{3,141}=2.99, p=$ 
Figure 3. Face memory using the continuous measure from 1 ("Definitely did not see this face last week") to 9 ("Definitely did see this face last week") with a midpoint of 5 ("I guessed randomly").

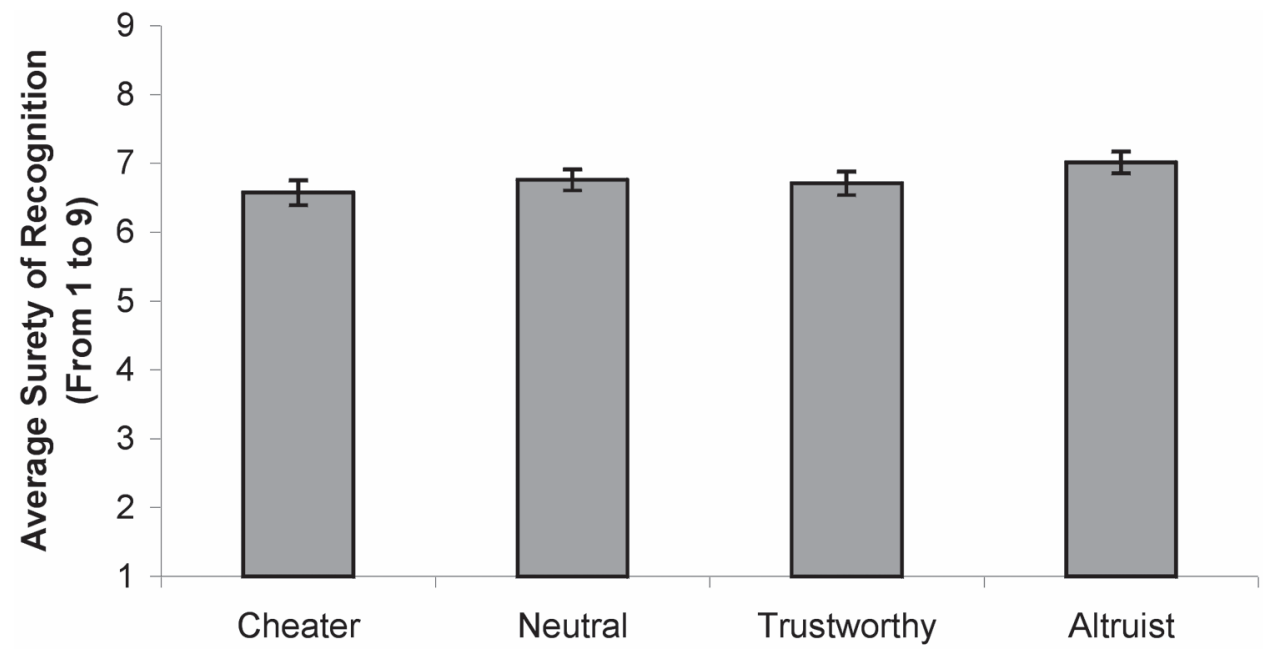

0.033: There was no effect of character within low-status descriptions, $F_{3,141}=$ 0.34 , n.s., but there was an effect within high-status descriptions, $F_{3,141}=4.88, p=$ 0.003 , in that high-status altruists (mean $=3.1$ out of 5 , s.e. $=0.20$ ) were remembered better than high-status cheaters (mean $=2.3$, s.e. $=0.20), F_{1,47}=17.87, p<$ 0.001 ; high-status trustworthy individuals (mean $=2.6$, s.e. $=0.25), F_{1,47}=4.09, p$ $=0.049$; and high-status neutrals (mean $=2.4$, s.e. $=0.22), F_{1,47}=9.60, p=0.003$.

\section{Discussion}

In this experiment the pictures and descriptions were presented on the same medium and participants could examine the stimuli at their own pace. Pictures paired with descriptions of altruistic behavior were seen as more attractive, and pictures paired with descriptions of trustworthiness were looked at longer. As in Experiment One, there was no tendency for participants to differentially remember faces paired with descriptions of cheating behavior. However, and contrary to Experiment One, there was a tendency for faces paired with descriptions of altruism by high-status individuals to be more confidently remembered.

\section{GENERAL DISCUSSION}

Overall, the two experiments failed to replicate Mealey et al.'s (1996) results. As we discussed earlier, it is possible that Mealey et al.'s apparent special memory for cheaters of low status (observed more strongly among male participants) was an artifact of the differential salience of the descriptions used in their study. It remains 
possible, of course, that the fault resides in the character descriptions used in this study. For instance, our neutral descriptions were not necessarily neutral, as they positively affected the attractiveness ratings of the faces with which they were paired. There was no relationship, however, between the degree of change in attractiveness as a result of reading the descriptions and the recognition of faces. Similarly, there was no relationship between the intensity of cheating in the cheating descriptions and the likelihood of them being remembered one week later.

One potentially interesting result from the second experiment is that high-status altruistic individuals were more confidently remembered (as measured on a continuous scale), supporting Brown and Moore's (2000) idea that detecting altruists and cooperating only with them is one way to solve the problem of exploitation in reciprocal social exchange. This finding was not obtained, however, in Experiment One, and should thus be interpreted with caution. Nevertheless, it might be worthwhile to attempt to replicate this finding and to further examine biased memory for successful altruists.

Returning to cheater recognition, our inability to replicate Mealey et al.'s (1996) results suggests that either cheater recognition is not nearly as robust as previously believed, or the observed cheater recognition was largely an artifact of the materials used to study it. The results of the present study, along with those of Baron and Burnstein's (2002), Oda's (1997), and Chiappe et al.'s (2004), provide equivocal support for biased memory for cheaters. This does not necessarily mean that cheaters may not look different from cooperators, as found by Brown, Palameta, and Moore (2003), and Yamagishi et al. (2003), or that character information does not alter recognition. What it does mean is that it is unclear whether knowing something about someone's cheating tendencies makes that person more memorable than someone else. Perhaps people's memory for cheaters or altruists depends on their frequencies in the population, such that cheaters are remembered in cooperative environments and altruists are remembered in noncooperative environments because they are rare. ${ }^{2}$ This could account for the differences between studies (although we find it unlikely that the McMaster student population contains a far greater proportion of cheaters than the College of Saint Benedict/Saint John's University student population), but would suggest a more general cognitive mechanism such as reputation tracking rather than specifically cheater recognition.

Our results should not discourage further attempts to detect special cheater recognition abilities. It is quite possible that the artificiality of our study did not promote the activation of cheater recognition mechanisms involved in social exchange. For instance, our ancestors were probably seldom exposed to a consecutive series of 40 new individuals within a very short period of time. Perhaps the relevant mechanisms were activated but became ineffectual because of cognitive load or low motivation. It may be worthwhile to perform a more ecologically valid experiment, such as Oda's (1997) experiment involving face recognition in the prisoner's dilemma game, but involving participants who actually play with real people. Such an experiment could use a task such as the public goods game (e.g., Fehr and Gächter 
2000), which is like a prisoner's dilemma and allows a continuum of responses rather than a dichotomous cooperate/defect decision.

We wish to thank Martin Daly, Grant Harris, Danny Krupp, Vernon Quinsey, Michael Seto, Margo Wilson, and three anonymous reviewers for helpful comments on the manuscript; Lisa DeBruine for writing the computer program for Experiment Two; Agnes Rekas for help designing the stimuli and collecting data; and Catherine Duquette for entering the data. The research was supported by grants from the Social Sciences and Humanities Research Council of Canada awarded to Martin Lalumière and Margo Wilson.

Pat Barclay received his Ph.D. from McMaster University, where he conducted research on altruism, costly signaling, and cooperative games. He is now a postdoctoral associate in the Department of Neurobiology and Behavior at Cornell University.

Martin Lalumière (Ph.D.) was a Research Psychologist at the Centre for Addiction and Mental Health and Associate Professor of Psychiatry and Criminology at the University of Toronto during the completion of this work. He is now Associate Professor in the Department of Psychology and Neuroscience at the University of Lethbridge, and his research interests include the causes of sexual aggression, the development of sexual preferences, and the nature of psychopathy.

\section{NOTES}

1. Examples in the sports area: "A.B. was caught using steroids at the World Swimming Championships when he was competing" (high-status cheater), "R.F. is a Zamboni driver who biked across the province on his own recently" (low-status neutral); "B.A. is a groundskeeper who makes sure his job is done well regardless of whether anyone will see" (low-status trustworthy); "L.B. was a professional basketball player who used to give some of his salary to youth programs in his neighborhood" (high-status altruist). The complete set of descriptions is available from the first author.

2. We thank an anonymous reviewer for this suggestion.

\section{REFERENCES}

Axelrod, R., and W. D. Hamilton

1981 The Evolution of Cooperation. Science 211:1390-1396.

Barclay, P.

2004 Trustworthiness and Competitive Altruism Can Also Solve the "Tragedy of the Commons." Evolution and Human Behavior 25:209-220.

Baron, A. S., and E. U. Burnstein

2002 Are Humans Equipped with a Specific Cheater-Detection Module or a More General Person-Impression Module? Evidence from a Face Recognition Experiment. Poster presented at the $14^{\text {th }}$ Annual Meeting of the Human Behavior and Evolution Society, Rutgers University, New Jersey.

Brown, W. M., and C. Moore

2000 Is Prospective Altruist-Detection an Evolved Solution to the Adaptive Problem of Subtle Cheating in Cooperative Ventures? Supportive Evidence Using the Wason Selection Task. Evolution and Human Behavior 21:25-37.

Brown, W. M., B. Palameta, and C. Moore

2003 Are There Nonverbal Cues to Commitment? An Exploratory Study Using the Zero-Acquaintance Video Presentation Paradigm. Evolutionary Psychology 1:42-69.

Chiappe, D., A. Brown, B. Dow, J. Koontz, M. Rodriguez, and K. McCulloch

2004 Cheaters Are Looked at Longer and Remembered Better Than Cooperators in Social Exchange Situations. Evolutionary Psychology 2:108-120. 
Cosmides, L.

1989 The Logic of Social Exchange: Has Natural Selection Shaped How Humans Reason? Studies with the Wason Selection Task. Cognition 31:187-276.

Cosmides, L., and J. Tooby

1992 Cognitive Adaptations for Social Exchange. In The Adapted Mind, J. Barkow, L. Cosmides, and J. Tooby, eds. Pp.163-228. New York: Oxford University Press.

Cummins, D. D.

1999 Cheater Detection Is Modified by Social Rank: The Impact of Dominance on the Evolution of Cognitive Functions. Evolution and Human Behavior 20:229-248.

Fehr, E., and S. Gächter

2000 Cooperation and Punishment in Public Goods Experiments. American Economic Review 90:980-994.

Fodor, J.

2000 Why We Are So Good at Catching Cheaters. Cognition 75:29-32.

Gigerenzer, G., and K. Hug

1992 Domain-Specific Reasoning: Social Contracts, Cheating, and Perspective Change. Cognition 43:127-171.

Hare, R. D.

1991 Manual for the Revised Psychopathy Checklist. Toronto: Multi-Health Systems.

Hare, R. D., A. E. Forth, and K. E. Strachan

1992 Psychopathy and Crime across the Life Span. In Aggression and Violence throughout the Life Span, R. D. Peters, R. J. McMahon, and V. L. Quinsey, eds. Pp. 285-300. London: Sage.

Harris, G. T., M. E. Rice, and V. L. Quinsey

1994 Psychopathy as a Taxon: Evidence That Psychopaths Are a Discrete Class. Journal of Consulting and Clinical Psychology 62:387-397.

Kiyonari, T., S. Tanida, and T. Yamagishi

2000 Social Exchange and Reciprocity: Confusion or a Heuristic? Evolution and Human Behavior 21:411-427.

Lalumière, M. L., G. T. Harris, and M. E. Rice

2001 Psychopathy and Developmental Instability. Evolution and Human Behavior 22:75-92.

Lalumière, M. L., and V. L. Quinsey

1996 Sexual Deviance, Antisociality, Mating Effort, and the Use of Sexually Coercive Behaviors. Personality and Individual Differences 21:33-48.

Levenson, M. R., K. A. Kiehl, and C. M. Fitzpatrick

1995 Assessing Psychopathic Attributes in a Noninstitutionalized Population. Journal of Personality and Social Psychology 1:151-158.

Mealey, L.

1995 The Sociobiology of Sociopathy: An Integrated Evolutionary Model. Behavioral and Brain Sciences 18:523-599.

Mealey, L., C. Daood, and M. Krage

1996 Enhanced Memory for Faces of Cheaters. Ethology and Sociobiology 17:119-128.

Oda, R.

1997 Biased Face Recognition in the Prisoner's Dilemma Game. Evolution and Human Behavior 18:309-315.

Seto, M. C., N. A. Khattar, M. L. Lalumière, and V. L. Quinsey

1997 Deception and Sexual Strategy in Psychopathy. Personality and Individual Differences 22:301-307.

Smith, E. A., S. Bowles, and H. Gintis

2000 Cooperation and Costly Signaling. Journal of Theoretical Biology 213:103-119.

Trivers, R. L.

1971 The Evolution of Reciprocal Altruism. Quarterly Review of Biology 46:35-57.

Yamagishi, T., S. Tanida, R. Mashima, E. Shimoma, and S. Kanazawa

2003 You Can Judge a Book by Its Cover: Evidence That Cheaters May Look Different from Cooperators. Evolution and Human Behavior 24:290-301.

Zahavi, A., and A. Zahavi

1997 The Handicap Principle: A Missing Piece of Darwin's Puzzle. New York: Oxford University Press. 